Original Article

\title{
COMPARATIVE STUDY BETWEEN PERIOSTIN AND OSTEOCALCIN AS BIOMARKERS FOR OSTEOPOROSIS AND FRACTURE RISK IN EGYPTIAN POSTMENOPAUSAL WOMEN
}

\author{
ALAA SALAM MOHAMED ${ }^{1}$, ASHRAF ISMAIL KHALIFA ${ }^{2}$, ASHRAF ABDEL-MONEAM ABOTALEB ${ }^{3}$, NOHA ABDEL- \\ RAHMAN ELDESOKY 4
}

${ }^{1}$ Biochemistry Department, Faculty of Pharmacy (Girls), Al-Azhar University, Cairo, Egypt, ${ }^{2}$ Rheumatology and Rehabilitation Department, Faculty of Medicine (Boys), Al-Azhar University, Cairo, Egypt, ${ }^{3}$ Clinical Pathology Department (Boys), Al-Azhar University, Cairo, Egypt, ${ }^{4}$ Biochemistry Department, Faculty of Pharmacy (Girls), Al-Azhar University, Cairo, Egypt

Email: alaa.salam2012.as@gmail.com

Received: 05 May 2020, Revised and Accepted: 04 Jul 2020

ABSTRACT

Objective: This study aimed to compare between periostin and osteocalcin as biomarkers in Egyptian postmenopausal women with osteoporosis and to explore their possible relationship with fracture risk.

Methods: This study included 90 postmenopausal females recruited from Al-Hussein University Hospital, Cairo, Egypt; divided into three groups; 35 postmenopausal osteoporotic females with low fracture risk (group I), 35 postmenopausal osteoporotic females with high fracture risk (group II), and 20 apparently healthy controls. Serum periostin, osteocalcin, and estrogen were measured by Enzyme Linked Immunosorbent Assay (ELISA). Fracture risk assessment was calculated. Alkaline phosphatase (ALP), total and ionized calcium, Aspartate transaminase (AST), and Alanine transaminase (ALT) were measured spectrophotometrically.

Results: The diagnostic performance of periostin for discriminating high fracture risk from low fracture risk groups showed the specificity of (68.6 \%) and sensitivity of $(100 \%)$, while for osteocalcin the specificity was (51.4\%) and the sensitivity was (68.6 \%) respectively. Moreover, the multi Receiver Operating Characteristics (multi-ROC) curve for periostin and osteocalcin together revealed improved specificity and sensitivity of (100 \%) each.

Conclusion: Periostin was superior to osteocalcin in discriminating high fracture risk from low fracture risk postmenopausal osteoporotic groups. Moreover, dual use of both markers gave the highest discriminative power between low and high fracture risk groups with $100 \%$ specificity and sensitivity.

Keywords: Postmenopausal osteoporosis, Periostin, Osteocalcin, Estrogen, DXA, Fracture risk

(C) 2020 The Authors. Published by Innovare Academic Sciences Pvt Ltd. This is an open access article under the CC BY license (http://creativecommons.org/licenses/by/4.0/) DOI: http://dx.doi.org/10.22159/ijpps.2020v12i9.38713. Journal homepage: https://innovareacademics.in/journals/index.php/ijpps.

\section{INTRODUCTION}

Osteoporosis is a skeletal metabolic disorder characterized by micro-architectural deterioration of bone tissue, this leads to an increase in bone fragility and fracture risk [1]. It is the most common metabolic bone disorder worldwide [2]. It was estimated that nearly 1 in each 2 postmenopausal females above the age of 50 will suffer a fragility fracture at some point in their life-time [3].

Bone strength is a measure of the resistance to bone fracture, which is determined by a collection of many skeletal characteristics including: composition, microarchitecture, size, and shape [4]. Dualenergy X-ray absorptiometry has been used widely for BMD measurement, bone strength assessment, and diagnosis of osteoporosis [5]. However, there are some limitations of DXA important to be considered. Bone mineral density (BMD) can be affected by positioning errors or artifacts, including osteoarthritis, fractures, and jewelry [6]. There are also many other factors that could affect DXA results, including recently administered gastrointestinal contrast or radionuclides, implants, devices, or any foreign material in the measurement area, and pregnancy [7]. Moreover, alkaline phosphatase (ALP) that has been usually used as a biomarker for osteoporosis, isn't specific for bone, and originates from different organs as liver and kidney [8].

Bone turnover biomarkers usually result from the bone remodeling process and can be measured in urine or serum [9]. They are released throughout life to repair microfractures in bone and to maintain mineral homeostasis [10]. They are classified as markers of bone formation as total alkaline phosphatase, bone-specific alkaline phosphatase, osteocalcin, and procollagen type $1 \mathrm{~N}$-terminal propeptide; and markers of bone resorption as hydroxyproline, deoxypyridinoline and pyridinoline [11].
Osteocalcin (OC) is a small protein (consists of 49 amino acids) encoded by the BGLAP gene synthesized by osteoblasts. The serum concentration of total OC has been considered a biochemical marker of osteogenesis that reflects the number and activity of osteoblasts [12]. It is the major and most thoroughly characterized bone-specific non collagenous protein in bone extracellular matrix that has been conserved in bone through evolution. It has a high affinity for calcium and plays an important role in matrix mineralization $[13,14]$.

Periostin, also named osteoblast-specific factor (OSF-2), is encoded by POSTN gene. It is an extracellular matrix protein of 836 amino acids with a molecular weight of approximately $93 \mathrm{kDa}$ [15]. Periostin exists in the basement membrane and lung's mesenchymal tissues. Its isomers are found also in the myocardium, skeletal muscle, heart valves, tendons, skin, periodontal ligaments, bones, and neoplastic tissues [16]. It is expressed predominantly in the periosteum, which covers the majority of bones and plays a vital role in regulating bone metabolism [17].

Periostin deficiency was related to osteoporosis and reduced bone strength [18]. The relationship between serum periostin, osteoporosis, and fracture risk in postmenopausal females is still unclear. Hence, the present work studies the possible relationship between serum periostin, BMD, estrogen, and fracture risk in Egyptian postmenopausal females compared to healthy postmenopausal controls. Moreover, the present study compares between periostin and osteocalcin performance as osteoporosis markers.

\section{MATERIALS AND METHODS}

\section{Study population}

This study was conducted on 90 postmenopausal females with age range (50-62) years old), divided into 70 osteoporotic females 
recruited from Rheumatology and Rehabilitation Department, AlHussein University Hospital, Cairo, Egypt in the period from December 2016 till March 2018, and 20 healthy postmenopausal volunteers taken as control group. Osteoporotic women were categorized into two groups; 35 with low fracture risk (group I) and 35 with high fracture risk (group II) according to the American Association of Clinical Endocrinologists (AACE) recommendations [19]. The present study conforms to recognized standards including Declaration of Helsinki, US Federal Policy for the Protection of Human Subjects, and European Medicines Agency Guidelines for Good Clinical Practice; and was approved by Research Ethical Committee of Faculty of Pharmacy, girls, Al-Azhar University (REC number: 252). Written consents were taken from every participant prior to their enrollment in the study. Also, all participants have given written informed consent for publication.

\section{Inclusion criteria}

1) Inclusion criteria for high fracture risk patients:

a) Postmenopausal females with BMD T-score of-2.5 or below at spine and hip.

b) Postmenopausal females with BMD T-score- 1 to- 2.5 at hip or spine with FRAX® 10-year probability for major osteoporotic fracture $\geq 20 \%$ or the 10 -year probability of hip fracture $\geq 3 \%$.

\section{2) Inclusion criteria for low fracture risk patients:}

Postmenopausal females with BMD T-score-1 to-2.5 at hip or spine with FRAX® 10-year probability for major osteoporotic fracture $<20$ $\%$ or hip fracture $<3 \%[19,20]$.

3) Inclusion criteria for control group

Postmenopausal females with BMD T-score of more than-1.

\section{Exclusion criteria}

1. Premenopausal and postmenopausal females with age more than $62 \mathrm{y}$.

2. Chronic diseases (Renal, Liver, Pulmonary, Cardiovascular, or any other major illness that could affect parameters.

\section{Patient taking medications for osteoporosis.}

4. Women who previously undergone a hysterectomy in young age.

All participants were subjected to physical examination, full clinical examination with particular attention if there were low back pain, pain in spine, forearm or femur, and DXA scan to assess BMD. Assessment of serum calcium levels (total and ionized) and alkaline phosphatase were done by colorimetric methods. Liver function tests, including ALT and AST were done by the kinetic ultraviolet method using (Biolis50i Superior, Japan) to ensure that any rise in ALP levels originate from bone. Osteocalcin and periostin were estimated by ELISA (Tecan A-5082, Austria). Estrogen was assessed by ELISA (Biotek, Japan).

\section{Samples collection}

Blood samples $(8 \mathrm{ml})$ were collected by trained laboratory technicians under complete aseptic conditions and allowed to clot for $30 \mathrm{~min}$ then centrifuged at $3000 \mathrm{rpm}$ for $15 \mathrm{~min}$. The serum was aspirated and divided into three aliquots, kept at- $80{ }^{\circ} \mathrm{C}$ until an assessment of calcium (Ca), alkaline phosphatase (ALP), alanine transaminase (ALT), aspartate transaminase (AST), estrogen (E2), osteocalcin (OC), and periostin.

\section{Statistical analysis}

The Statistical Package for the Social Sciences (SPSS) (V. 24.0, IBM Corp., USA, 2016) was used for data analysis. Date was expressed as Median and Percentiles for quantitative nonparametric measures.

The following tests were used; Wilcoxon Rank Sum was used for comparison between two independent groups, Kruskall Wallis was used for comparison between more than 2 patient groups, and Ranked Spearman correlation was used to study the possible association between two variables. The probability of error at 0.05 or less was considered significant, while at 0.01 and 0.001 was considered highly significant.

The Receiver Operating Characteristics (ROC) curves were constructed to obtain the most sensitive and specific cutoff values for each marker. Area under the curve (AUC) was calculated.

\section{RESULTS}

The Demographic data, clinical characters, and biochemical parameters of the control group, osteoporotic patients with low fracture risk (group I) and with high fracture risk (group II) were presented in table 1 . Weight, body mass index and ALT were significantly increased in group I and group II compared to control group at ${ }^{* *} \mathrm{p} \leq 0.001$ and ${ }^{* *} \mathrm{p} \leq 0.01$, respectively. Total calcium and ionized calcium showed a significant decrease in group I and group II compared to control group (** $\leq 0.001)$, while osteocalcin, ALP, and periostin showed a significant increase in group I and group II compared to control group $(* * p \leq 0.001)$. Moreover, spine BMD Tscore and total hip BMD T-score showed a significant decrease, while major osteoporotic (FRAX) (\%) and hip fracture (FRAX) (\%) showed significant increase in group I and group II compared to the control group $(* * p \leq 0.001)$, as mentioned previously in inclusion criteria.

Table 1: Demographic, clinical, and biochemical parameters of all studied groups

\begin{tabular}{lllll}
\hline Variables & Control (n= 20) & Group I (n = 35) & Group II (n = 35) & p value \\
\hline Age (Years) (25Perc-75perc) & $57.5(50.25-60.75)$ & $58.1(53-59)$ & $60(57-62)$ & 0.213 \\
Height (Cm) (25Perc-75perc) & $156(153-158.75)$ & $155(151-159)$ & $157(149-160)$ & 0.906 \\
Weight (Kg) (25Perc-75perc) & $64(60-72.25)$ & $77^{\mathrm{a}}(69-85)$ & $80^{\mathrm{a}}(70-97)$ & $0.001^{* *}$ \\
BMI (Kg/m) (25Perc-75perc) & $26.85(24.55-30.55)$ & $32.4^{\mathrm{a}}(29.1-37.3)$ & $35^{\mathrm{a}}(27.3-39.3)$ & $0.001^{* *}$ \\
ALT (U/l) (25Perc-75perc) & $6(4-7)$ & $7(4-12)$ & $11^{\mathrm{a}}(6-14)$ & $0.016^{* *}$ \\
AST (U/l) (25Perc-75perc) & $6.5(4.25-9)$ & $9(6-13)$ & $9(5-14)$ & 0.135 \\
Total Ca (mg/dl) (25Perc-75perc) & $9.79(9.6-10.2175)$ & $6.04^{\mathrm{a}}(5.4-7.03)$ & $5.48^{\mathrm{a}, \mathrm{b}}(5.06-6.23)$ & $0^{* *}$ \\
Ionized Ca (mg/dl) (25Perc-75perc) & $4.62(4.5925-4.6875)$ & $4.59(4.18-4.9)$ & $4.18^{\mathrm{a}, \mathrm{b}}(3.95-4.58)$ & $0^{* *}$ \\
ALP (U/l) (25Perc-75perc) & $108(98.25-116.25)$ & $350^{\mathrm{a}}(315-369)$ & $454^{\mathrm{a}, \mathrm{b}}(409-472)$ & $0^{* *}$ \\
Spine BMD T-score (25Perc-75perc) & $-0.1(-0.3-0.675)$ & $-2^{\mathrm{a}}(-2.8-(-1.1))$ & $-2.2^{\mathrm{a}}(-2.7-(-0.3))$ & $0^{* *}$ \\
Total hip BMD T-score (25Perc-75perc) & $0.45(0-1.075)$ & $-1.7^{\mathrm{a}}(-2-(-1.4))$ & $-2.3^{\mathrm{a}, \mathrm{b}}(-2.4-(-1.4))$ & $0^{* *}$ \\
Major osteoporotic (FRAX) (\%) (25Perc-75perc) & $2.2(1.6-2.75)$ & $6.3^{\mathrm{a}}(4.2-8.7)$ & $15^{\mathrm{a}, \mathrm{b}}(11.9-18)$ & $0^{* *}$ \\
Hip fracture (FRAX) (\%) (25Perc-75perc) & $0.1(0-0.1)$ & $1.1^{\mathrm{a}}(0.9-1.9)$ & $5^{\mathrm{a}, \mathrm{b}}(3.9-6.4)$ & $0^{* *}$ \\
Estrogen (pg/ml) (25Perc-75perc) & $5.5(3.25-8)$ & $5(3-10)$ & $6(3-11)$ & 0.434 \\
Osteocalcin (ng/ml) (25Perc-75perc) & $7.15(5.4-11.125)$ & $26.2^{\mathrm{a}}(23.9-29)$ & $27.7^{\mathrm{a}}(25.5-30)$ & $0^{* *}$ \\
Periostin (ng/ml) (25Perc-75perc) & $483(411.75-564.75)$ & $817.5^{\mathrm{a}}(710-886)$ & $1102^{\mathrm{a}, \mathrm{b}}(915-1340)$ & $0^{* *}$ \\
\hline
\end{tabular}

a: Significant difference from control group, b: Significant difference from group I, ${ }^{*} \mathrm{p} \leq 0.05$ was considered significant, ${ }^{* *} \mathrm{p} \leq 0.01$ and 0.001 were considered highly significant.

Correlation of osteocalcin with the other studied parameters revealed positive significant correlation with ALP in group I and group II $(\mathrm{r}=$ $\left.0.898,{ }^{* *} \mathrm{p} \leq 0.001\right)$ and $\left(\mathrm{r}=0.938,{ }^{* *} \mathrm{p} \leq 0.001\right)$ respectively. There were also positive significant correlations between osteocalcin and each of major osteoporotic (FRAX) (\%) and hip fracture (FRAX) (\%) in group II $\left(\mathrm{r}=0.791,{ }^{* *} \mathrm{p} \leq 0.001\right)$ and $\left(\mathrm{r}=0.922,{ }^{* *} \mathrm{p} \leq 0.001\right)$ respectively (table 2$)$. 
Table 2: Correlation of osteocalcin $(\mathrm{ng} / \mathrm{ml})$ with all studied parameters in controls, group I, and group II

\begin{tabular}{|c|c|c|c|c|c|c|}
\hline \multirow[t]{3}{*}{ Studied parameters } & \multicolumn{6}{|c|}{ Correlation of osteocalcin (ng/ml) } \\
\hline & \multicolumn{2}{|c|}{ Control $(n=20)$} & \multicolumn{2}{|c|}{ Group I (n = 35) } & \multicolumn{2}{|c|}{ Group II (n= 35) } \\
\hline & r-value & p value & r-value & p value & r-value & p value \\
\hline Total Ca (mg/dl) & 0.212 & 0.369 & -0.128 & 0.465 & -0.054 & 0.757 \\
\hline Ionized Ca (mg/dl) & 0.132 & 0.579 & -0.135 & 0.438 & -0.235 & 0.175 \\
\hline $\operatorname{ALP}(\mathrm{U} / \mathrm{l})$ & 0.108 & 0.651 & 0.898 & $0^{* *}$ & 0.979 & $0 * *$ \\
\hline Spine BMD T-score & -0.289 & 0.216 & -0.092 & 0.6 & -0.266 & 0.192 \\
\hline Total hip BMD T-score & -0.443 & 0.051 & -0.07 & 0.69 & -0.249 & 0.15 \\
\hline Major osteoporotic (FRAX) (\%) & 0.681 & $0.001^{* *}$ & 0.09 & 0.607 & 0.791 & $0^{* *}$ \\
\hline Hip fracture (FRAX) (\%) & 0.601 & $0.005^{*}$ & 0.113 & 0.518 & 0.922 & $0^{* *}$ \\
\hline Estrogen $(\mathrm{pg} / \mathrm{ml})$ & -0.45 & $0.046^{*}$ & -0.342 & $0.045^{*}$ & -0.066 & 0.706 \\
\hline
\end{tabular}

${ }^{*} \mathrm{p} \leq 0.05$ was considered significant, ${ }^{* *} \mathrm{p} \leq 0.01$ and 0.001 were considered highly significant.

The correlation between periostin and the other studied parameters revealed positive significant correlation between periostin and ALP in group I and group II (r-value $\left.=0.952,{ }^{* *} \mathrm{p} \leq 0.001\right)$ and $(\mathrm{r}$-value $=$ $\left.0.992,{ }^{* *} \mathrm{p} \leq 0.001\right)$ respectively. There were also positive significant correlations between periostin and each of major osteoporotic (FRAX) (\%) and hip fracture (FRAX) (\%) in group II (r-value $=0.754$,

\begin{abstract}
$\left.{ }^{* *} \mathrm{p} \leq 0.001\right)$ and ( $\mathrm{r}$-value $\left.=0.886,{ }^{* *} \mathrm{p} \leq 0.001\right)$ respectively and negative significant correlation with estrogen in controls and group I ( $\mathrm{r}$-value $\left.=-0.648,{ }^{* *} \mathrm{p} \leq 0.01\right)$ and (r-value $=-0.356,{ }^{* *} \mathrm{p} \leq 0.05$ ) respectively. Moreover, periostin showed positive significant correlation with osteocalcin in groups I and II ( $\mathrm{r}$-value $=0.938,{ }^{* *} \mathrm{p} \leq$ 0.001 ) and ( $\mathrm{r}$-value $=0.958,{ }^{* *} \mathrm{p} \leq 0.001$ ) respectively (table 3 ).
\end{abstract}

Table 3: Correlation of periostin $(\mathrm{ng} / \mathrm{ml})$ with all studied parameters in controls, group I, and group II

\begin{tabular}{|c|c|c|c|c|c|c|}
\hline \multirow[t]{3}{*}{ Studied parameters } & \multicolumn{6}{|c|}{ Correlation of periostin (ng/ml) } \\
\hline & \multicolumn{2}{|c|}{ Control $(n=20)$} & \multicolumn{2}{|c|}{ Group I (n = 35) } & \multicolumn{2}{|c|}{ Group II (n = 35) } \\
\hline & r-value & p value & r-value & p value & r-value & p value \\
\hline Total Ca (mg/dl) & -0.239 & 0.311 & -0.173 & 0.32 & -0.141 & 0.419 \\
\hline Ionized Ca (mg/dl) & -0.071 & 0.768 & -0.194 & 0.265 & -0.076 & 0.663 \\
\hline $\operatorname{ALP}(\mathrm{U} / \mathrm{l})$ & 0.02 & 0.935 & 0.952 & $0^{* *}$ & 0.992 & $0^{* *}$ \\
\hline Spine BMD T-score & -0.042 & 0.859 & -0.04 & 0.82 & -0.284 & 0.099 \\
\hline Total hip BMD T-score & 0.058 & 0.809 & -0.003 & 0.986 & -0.021 & 0.227 \\
\hline Major osteoporotic (FRAX) (\%) & 0.406 & 0.075 & 0.05 & 0.774 & 0.754 & $0^{* *}$ \\
\hline Hip fracture (FRAX) (\%) & 0.433 & 0.057 & 0.074 & 0.672 & 0.886 & $0 * *$ \\
\hline Estrogen $(\mathrm{pg} / \mathrm{ml})$ & -0.648 & $0.002^{* *}$ & -0.356 & $0.036^{*}$ & -0.25 & 0.978 \\
\hline Osteocalcin (ng/ml) & 0.406 & 0.075 & 0.938 & $0 * *$ & 0.958 & $0 * *$ \\
\hline
\end{tabular}

${ }^{*} \mathrm{p} \leq 0.05$ was considered significant, ${ }^{* *} \mathrm{p} \leq 0.01$ and 0.001 were considered highly significant.

Table (4) and (fig. 1) represents the output data of the receiver operating characteristics (ROC) curve for each of serum periostin and osteocalcin. At cut off 602 for periostin, the specificity and sensitivity were (68.6\% and $100 \%)$ respectively. At cut off 20.7 for osteocalcin, the specificity and sensitivity were (51.4\% and $68.6 \%$ ) respectively. Multi-ROC curve showed a huge improvement in the discriminative power of periostin and osteocalcin when used together as the sensitivity and specificity raised to $100 \%$, at cut off value of 850 for periostin.

Table 4: The discriminative power of serum periostin $(\mathrm{ng} / \mathrm{ml})$, osteocalcin $(\mathrm{ng} / \mathrm{ml})$, and combined (periostin/osteocalcin) between group I and group II osteoporotic patients

\begin{tabular}{|c|c|c|c|c|c|}
\hline Variable & Cutoff & AUC & \% Sensitivity & $\%$ Specificity & \% Efficacy \\
\hline Periostin (ng/ml) & 850 & 1.000 & 100.0 & 68.6 & 84.3 \\
\hline Osteocalcin (ng/ml) & 26.2 & 0.995 & 68.6 & 51.4 & 60 \\
\hline \multicolumn{6}{|c|}{ Multi-ROC: for periostin $(\mathrm{ng} / \mathrm{ml})$ at 850} \\
\hline Osteocalcin (ng/ml) & 32.5 & 1 & 100.0 & 100.0 & 100.0 \\
\hline
\end{tabular}

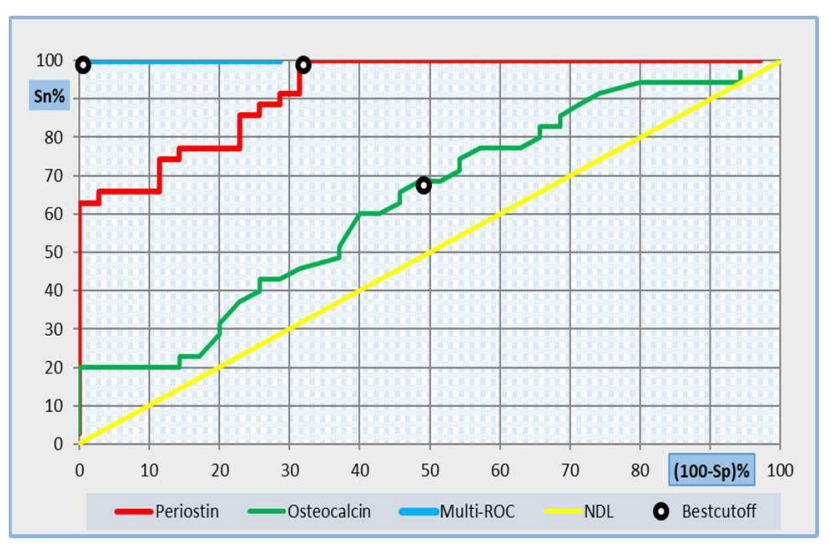

Fig. 1: ROC curve for discriminating patients with high fracture risk from those with low fracture risk showing the diagnostic performance of periostin $(\mathrm{ng} / \mathrm{ml})$, osteocalcin $(\mathrm{ng} / \mathrm{ml})$, and multi ROC for their combination 


\section{DISCUSSION}

Osteoporosis is usually caused by altered bone micro-structure predisposing patients to fragility and fractures [21]. Introducing a biomarker that could predict the risk of bone fracture would help in the early therapeutic intervention, reducing future fractures, and complications.

In the current study, there were reductions in spine BMD T-score, total hip BMD T-score, and serum $\mathrm{Ca}$ in group I and group II compared to the control group $\left({ }^{* *} \mathrm{p} \leq 0.001\right)$. This was matched with a study done by Tian et al. [22] who reported lower serum Ca and BMD in postmenopausal osteoporotic than non-osteoporotic females. Moreover, Qu et al. found lower BMD in the fracture group than in the non-fracture group; and in elder females than younger females. They stated that the risk of fracture increases with the reduction in bone density [23].

In the present study, major osteoporotic (FRAX) (\%) and hip fracture (FRAX) (\%) showed significant increases in group I and group II compared to control group. This result was similar to Tomasevic et al. who concluded that osteoporosis patients had a high risk of Major osteoporotic (FRAX) and hip fracture (FRAX) $\%$. They indicated that patients suffering from osteoporosis and who had a history of fractures had higher fracture risk in comparison to those suffering from osteopenia without history of fractures [24].

In the present work, osteocalcin showed a significant increase in both patients' groups compared to the control group at ${ }^{* *} \mathrm{p} \leq 0.001$ while no significant difference was obtained between groups I and II. This was matched with a study done by Alam et al. who reported a significant increase of osteocalcin in postmenopausal osteoporotic patients and explained it by accelerated osteoclastic activity due to the sudden depletion of estrogen which increases bone resorption on the expense of bone formation that is reflected in serum as increased osteocalcin levels [25].

Beg et al. reported that serum osteocalcin was significantly higher in postmenopausal females with osteoporosis than without $\left({ }^{*} \mathrm{p}<0.05\right)$. They reported reduced osteocalcin levels after treatment with risedronate, an osteoporosis medication; and concluded that osteocalcin can be potentially useful in the diagnosis and the monitoring of response to therapy in osteoporotic patients [26].

Singh et al. recommended the use of serum osteocalcin level as a screening tool for osteoporosis in postmenopausal females and advised only subjects having osteocalcin levels beyond osteocalcin cutoff point for DXA scan to grade the severity of osteoporosis [27].

Soroosh et al. discussed the relation between osteocalcin and bone formation by the following; Osteocalcin is produced by osteoblasts during bone formation process and binds to the c-carboxyglutamic acid (Gla) residues by its high affinity for calcium. This promotes the absorption of calcium to the hydroxyapatite in bone matrix and aids mineralization of bone. Decreases in bone mineralization (decreased hydroxyapatite crystal formation) in osteoporosis causes free osteocalcin to circulate in the blood and hence results in increased serum osteocalcin levels [28].

On the contrary, Rai et al. found very low levels of serum osteocalcin in postmenopausal females with fractures compared to the premenopausal females and linked it with reduced bone formation and increased resorption activity at late menopause [29].

Moreover, Liu et al. found no significant difference of the pooled serum osteocalcin in postmenopausal osteoporotic patients in comparison with postmenopausal controls. They recommended not to use serum osteocalcin as indicator for high bone turnover status in postmenopausal females unless new techniques for standardized circulatory osteocalcin evaluation are introduced in the future, since osteocalcin molecules are quite heterogeneous (different fragments and different carboxylation status) in the circulation, and can be influenced by many metabolic events [30].

The previously discussed controversy demonstrates that osteocalcin could not be considered as a reliable marker for osteoporosis diagnosis and monitoring; hence it appears the necessity of searching for new biomarkers.

Regarding the correlation of osteocalcin with other biomarkers, there were significant positive correlations with ALP in group I and group II. This was compatible with the results obtained by Singh et al. who stated that alkaline phosphatase had a strong positive correlation with serum osteocalcin level [27].

In the present study, there were no significant correlations between osteocalcin and each of (total hip BMD T-score and spine BMD Tscore) in group I and group II. This was in accordance with a study done by Soroosh et al. who stated that serum osteocalcin levels did not correlate significantly with BMD in postmenopausal osteoporosis females [28].

In the current study, there were significant positive correlations between osteocalcin and each of (major osteoporotic (FRAX) \% and hip fracture (FRAX) \%) in group II. This was matched with Dai et al. who found a dose-dependent positive relationship between osteocalcin and the risk of hip fracture in Asian population [31].

In the current study, periostin showed a significant increase in both patients' groups compared to the control group at ${ }^{* *} \mathrm{p} \leq 0.001$ and a significant increase in high fracture risk group than the low fracture risk group at ${ }^{* *} \mathrm{p} \leq 0.001$. This means that high level of periostin was associated with an increased risk of fracture. These findings were agreed with a study done by Bonnet et al. who found periostin increased in females with incident fracture than those without [32]. Kim et al. and Sakellariou et al. found high serum periostin levels associated with increased fracture risk in postmenopausal females [33,34].

Varugheseet al. reported elevated serum periostin levels in response to bone injury and repair. They observed elevated periostin levels also in patients with radiological evidence of osteoporotic fracture [16].

Circulating periostin may reflect the adaptation of the metabolic activity of periosteum cells to the existing bone strains for maintenance of a stabilized bone quality. Women with lower bone mass and strength may have a higher mechanical strain in the remaining bone that would increase periostin expression. Thus, increased expression of periostin is reflected in serum by an increase of circulating periostin. However, at the level of bone, an increase of bone formation caused by an increased periostin expression is not enough to compensate the bone loss leading to fragility fractures [35].

De Lageneste et al. explained the role of periostin in bone regeneration by the activation of skeletal stem cells (SSCs) in the periosteum causing a high bone regenerative potential, reconstituting a pool of periosteal cells after injury [36]. Zhang et al. explained that mechanical activity and exercise may increase periostin production in osteoblasts, which in turn may inhibit the differentiation of osteoclasts by its effects on semaphorin-3A [37].

Kudo. explained that the cortical bone formation is regulated by the periostin-mediated blocking of random bone formation. They stated that, in response to mechanical stress, periostin expression is enhanced, and activates cellular functions to improve the irregular collagen fibrillogenesis and extracellular matrix organization to maintain tissue homeostasis [38].

Yan et al. found the initial levels of periostin after fracture significantly higher in osteoporotic patients than controls revealing that high periostin level was an independent predictor of femoral neck BMD in elderly females presenting with acute hip fracture. They declared that increased periostin levels during early healing phase may imply that periostin play a role in bone repair [39].

Luo and Deng. found no significant differences in serum periostin levels between postmenopausal females with normal and abnormal BMD Tscore, and reported that periostin is not a predictor of early-stage bone deterioration in Chinese postmenopausal females. However, during the course of their study, BMD data at one year after baseline indicated that the femur neck bone mineral content (BMC) and T-score became lower in women with higher baseline serum periostin [40]. This indicates a powerful relation of periostin and BMD. 
In the present work, no significant correlations were obtained between periostin and bone mineral density in the three groups. This result is compatible with Walsh et al. who reported that there were no significant correlations between serum periostin and BMD at the lumbar spine or total hip, when analyzed as a group and within each group [41].

However, Gossiel et al. revealed that the changes in periostin levels were positively correlated with the changes in total hip BMD and femoral neck BMD in postmenopausal females with osteoporosis after treatment with teriparatide [42].

In the present work, there was a significant positive correlation between periostin and each of (major osteoporotic (FRAX) (\%) and hip fracture (FRAX) (\%)) in group II. This was matched with Terpos et al. who stated that periostin was elevated in the bone marrow plasma and in the serum of newly diagnosed symptomatic multiple myeloma patients and correlates with extensive bone lytic lesions, bone fractures and extensive osteolysis [43].

Also, there were no significant correlations between periostin and each of (total hip BMD T-score and spine BMD T-score) in both patients' groups in the present study. This was as the findings of Rousseau et al. who reported that serum periostin was not significantly associated with BMD of the spine or the hip [44]. Yan et al. reported that serum periostin level was negatively correlated with femoral neck BMD, an acute hip fracture was associated with a transient change of serum periostin levels in older females, and that measurement of serum periostin around the time of bone healing phase may include assessment of response to fracture therapy [39].

In the current work, there was a significant positive correlation between periostin and ALP in group I and group II. This result is compatible with Anastasilakis et al. [45] and in contrast with $\mathrm{Hu}$ et al. [46].

In the current work, there was a significant positive correlation between osteocalcin and periostin in group I and group II. However, the diagnostic performance of periostin and osteocalcin in discrimination between group I and group II using ROC curves revealed a more powerful discriminating capability of periostin than osteocalcin. The best cutoff value of periostin was taken at $850 \mathrm{ng} / \mathrm{ml}$ with specificity and sensitivity (100\% and $68.6 \%$ ), respectively, while the best cutoff value of osteocalcin was taken at $26.2 \mathrm{ng} / \mathrm{ml}$ with specificity and sensitivity (68.6\% and $51.4 \%$ ) respectively. Moreover, a multi ROC curve was performed for periostin and osteocalcin together and revealed improved specificity and sensitivity of $100 \%$ for each at the cut off values (850 and 32.5), respectively.

\section{CONCLUSION}

The present study implies a potential role of periostin as a promising biomarker for the prediction of fracture risk in postmenopausal osteoporotic females. Moreover, periostin was superior to osteocalcin in discrimination of high fracture risk from low fracture risk patients. On top of that and according to the studied Egyptian population, Dual assessment of osteocalcin, and periostin seemed to be more efficient in identifying high fracture risk from low fracture risk osteoporosis patients than the use of each of them alone.

\section{LIMITATIONS AND RECOMMENDATIONS}

- The number of participants in the present study was the maximum number that authors could afford financially as this study was completely self-funded, so further studies are recommended on large scale to confirm our results.

- The present study involved only postmenopausal women so further studies are recommended to examine the role of periostin as a biomarker for senile osteoporosis in both sex groups and in different age groups.

\section{ACKNOWLEDGEMENT}

We would like to thank all staff members of the Rheumatology and Rehabilitation department and technicians of immunology department in Al-Hussein University Hospital for their great help in this research.

\section{FUNDING}

Nil

\section{AUTHORS CONTRIBUTIONS}

$\mathrm{AI}, \mathrm{AA}$ and NA were involved in paper idea, protocol development and data analysis.

AI and AS were responsible for patient recruitment and clinical records.

NA and AS were responsible for gaining ethical approval and writing the manuscript.

All authors reviewed and edited the manuscript and approved the final version of the manuscript.

\section{CONFLICTS OF INTERESTS}

None

\section{REFERENCES}

1. Hutomo DI, Masulili SLC, Tadjoedin FM, Kusdhany LS. Correlation of serum osteocalcin level and periodontal attachment loss with osteoporosis risk status in postmenopausal women. Int J Appl Pharm 2018;9:92-4.

2. Jawhar DS, Hassan NA, Shamssain MH. Dual-energy x-ray absorptiometry scan (DXA) findings in diabetic and nondiabetic female: a retrospective cohort study. Med J Malaysia 2020; 75:47-51.

3. Gunna SH, Yadav S. Low bone mass and its correlation in systemic sclerosis. Indian J Rheumatol 2019;14:100-1.

4. Diez Perez A, Brandi ML, Al-Daghri N, Branco JC, Bruyere O, Cavalli L, et al. Radiofrequency echographic multispectrometry for the in vivo assessment of bone strength: state of the art-outcomes of an expert consensus meeting organized by the European society for clinical and economic aspects of osteoporosis, osteoarthritis and musculoskeletal diseases (ESCEO). Aging Clin Exp Res 2019;31:1375-89.

5. Marushchak M, Krynytska I. Pharmacological treatment of osteoporosis in patients with coronary heart disease complicated plicated by chronic heart failure. Asian J Pharm Clin Res 2019;12:443-6.

6. Jain RK, Vokes T. Dual-energy X-ray absorptiometry. J Clin Densitom 2017;20:291-303.

7. Krugh M, Langaker MD. Dual-energy X-ray absorptiometry (DEXA). In: Stat pearls. Available from: https:// www.ncbi.nlm.nih.gov/books/NBK519042/. [Last accessed on 24 Apr 2020].

8. Brichacek AL, Brown CM. Alkaline phosphatase: a potential biomarker for stroke and implications for treatment. Metab Brain Dis 2019;34:3-19.

9. Abreu C, Dionisio MR, Silva JC, Costa L. Cancer treatmentinduced bone loss (CTIBL). In: Huhtaniemi I, Martini L. (editors). Encyclopedia of endocrine diseases. 2nd ed. United States: San Diego; 2018. p. 296-303.

10. Bhattoa HP. Laboratory aspects and clinical utility of bone turnover markers. EJIFCC 2018;29:117-28.

11. Rowe P, Koller A, Sharma S. Physiology, bone remodeling. Stat Pearls. Available from: https://www.ncbi.nlm.nih.gov/ books/NBK499863/. [Last accessed on 29 Mar 2020].

12. Diaz Franco MC, De Leon RFD, Villafan Bernal JR. Osteocalcin-GPRC6A: an update of its clinical and biological multi-organic interactions (review). Mol Med Rep 2019;19:15-22.

13. Macias I, Alcorta Sevillano N, Rodriguez CI, Rodriguez CI, Arantza Infante A. Osteoporosis and the potential of cell-based therapeutic strategies. Int J Mol Sci 2020;21:1653.

14. Carvalho MS, Poundarik AA, Cabral JMS, da Silva $\mathrm{CL}$, Vashishth D. Biomimetic matrices for rapidly forming mineralized bone tissue based on stem cell-mediated osteogenesis. Sci Rep 2018;8:14388.

15. Gonzalez Gonzalez L, Alonso J. Periostin: a matricellular protein with multiple functions in cancer development and progression. Front Oncol 2018;8:225.

16. Varughese R, Semprini R, Munro C, Fingleton J, Holweg $\mathrm{C}$, Weatherall $\mathrm{M}$, et al. Serum periostin levels following small 
bone fractures, long bone fractures and joint replacements: an observational study. Allergy Asthma Clin Immunol 2018;14:30.

17. Li C, Li X, Wang X, Miao P, Liu J, Li C, et al. Periostin mediates estrogen-induced osteogenic differentiation of bone marrow stromal cells in ovariectomized rats. BioMed Res Int 2020:110. https://doi.org/10.1155/2020/9405909.

18. Garnero P, Cremers S. Chapter 78-bone turnover markers. In: Bilezikian JP, Martin TJ, Clemens TL. et al. editors. Principles of Bone Biology. 4th ed. London: Academic Press; 2019. p. 1801-39.

19. Camacho PM, Petak SM, Binkley N, Clarke BL, Harris ST, Hurley $\mathrm{DL}$, et al. AACE/ACE clinical practice guidelines. American association of clinical endocrinologists. Clinical guidelines for the diagnosis and treatment of postmenopausal osteoporosis. Endocr Pract 2016;22:S1-42.

20. Tu KN, Lie JD, Wan CKV, Cameron M, Austel AG, Nguyen JK, et al. Osteoporosis: A review of treatment options. $\mathrm{P} \mathrm{T}$ J 2018;43:92-104.

21. Porter JL, Varacallo M. Osteoporosis. stat pearls. Available from: https://www.ncbi.nlm.nih.gov/books/NBK441901practice/. [Last accessed on 15 Mar 2020].

22. Tian L, Yang R, Wei L, Liu J, Yang Y, Shao F, et al. Prevalence of osteoporosis and related lifestyle and metabolic factors of postmenopausal women and elderly men: a cross-sectional study in Gansu province, Northwestern of China. Medicine (Baltimore) 2017;96:e8294.

23. Qu XL, Zheng B, Chen TY, Cao ZR, Qu B, Jiang T. Bone turnover markers and bone mineral density to predict osteoporotic fractures in older women: a retrospective comparative study. Orthopaedic Surgery 2020;12:116-23.

24. Tomasevic TS, Vazic A, Issaka A, Hanna F. Comparative assessment of fracture risk among osteoporosis and osteopenia patients: a cross-sectional study. Open Access Rheumatol 2018;10:61-6.

25. Alam MF, Rana MA, Alam MS. Osteocalcin, a promising marker of osteoporosis: evaluation in postmenopausal females with osteoporosis. Int Adv Med 2019;6:xxx-x.

26. Beg M, Akhtar N, Alam MF. Vitamin D status and serum osteocalcin levels in post-menopausal osteoporosis: effect of bisphosphonate therapy. JIACM 2014;15:172-6.

27. Singh S, Kumar D, Lal AK. Serum osteocalcin as a diagnostic biomarker for primary osteoporosis in women. J Clin Diagn Res 2015;9:RC04-7.

28. Soroosh M, Khabbazi A, Mahdavi AM. Serum osteocalcin levels in postmenopausal osteoporotic women receiving alendronate. Rheumatol Res 2018;3:83-9.

29. Rai M, Rai T, Rai S, Parinita K. Serum osteocalcin: a potential biomarker for primary osteoporosis. Int J Clin Biochem Res 2018;5:392-6.

30. Liu Z, Chen R, Jiang Y, Yang Y, He L, Luo C, et al. A meta-analysis of serum osteocalcin level in postmenopausal osteoporotic women compared to controls. BMC Musculoskeletal Disord 2019;20:532.

31. Dai Z, Wang R, Ang LW, Yuan JM, Koh WP. Bone turnover biomarkers and risk of osteoporotic hip fracture in an Asian population. Bone 2016;83:171-7.

32. Bonnet N, Biver E, Chevalley T, Rizzoli R, Garnero P, Ferrari S. Serum levels of a cathepsin-K generated periostin fragment predict incident low-trauma fractures in postmenopausal women independently of BMD and FRAX. JBMR 2017;32:2232-8.

33. Kim BJ, Rhee Y, Kim CH, Baek KH, Min YK, Kim DY. Plasma periostin associates significantly with non-vertebral but not vertebral fractures in postmenopausal women: clinical evidence for the different effects of periostin depending on the skeletal site. Bone 2015;81:435-41.

34. Sakellariou GT, Anastasilakis AD, Bisbinas I, Oikonomou D, Gerou S, Polyzos SA. Circulating periostin levels in patients with AS: association with clinical and radiographic variables, inflammatory markers and molecules involved in bone formation. Rheumatology (Oxford) 2015;54:908-14.

35. Kim BJ, Lee SH, Koh JM. Potential biomarkers to improve the prediction of osteoporotic fractures. Endocrinol Metab (Seoul) 2020;35:55-63.

36. De Lageneste OD, Julien A, Abou-Khalil R, Frangi G, Carvalho C, Cagnard $\mathrm{N}$, et al. Periostium contains skeletal stem cells with high bone regenerative potential controlled by periostin. Nat Commun 2018;9:773.

37. Zhang $M$, Ishikawa $S$, Inagawa $T N$, Ikemoto $H$, Guo $S$, Mand $\mathrm{S}$, et al. Influence of mechanical force on bone matrix proteins in ovariectomized mice and osteoblast-like MC3T3-E1 cells. In Vivo 2017;31:87-95.

38. Kudo A. Periostin in bone biology. In: Kudo A. editor. Periostin. Advances in Experimental Medicine and Biology. 1st ed. Singapore: Springer; 2019. p. 43-7.

39. Yan J, Liu HJ, Li H, Chen L, Bian YQ, Zhao B, et al. Circulating periostin levels increase in association with bone density loss and healing progression during the early phase of hip fracture in Chinese older women. Osteoporos 2017;28:2335-41.

40. Luo J, Deng W. Serum periostin level is not a predictor of earlystage bone loss in Chinese postmenopausal women. Clin Lab 2019;65. DOI:10.7754/clin.lab.2019.190329

41. Walsh JS, Gossiel F, Scott JR. Effect of age and gender on serum periostin: relationship to cortical measures, bone turnover and hormones. Bone 2017;99:8-13.

42. Gossiel F, Scott JR, Paggiosi MA, Naylor KE, Closkey EV, Peel NF, et al. The effect of teriparatide treatment on circulating periostin and its relationship to regulators of bone formation and BMD in postmenopausal women with osteoporosis. J Clin Endocrinol Metab 2018;103:1302-9.

43. Terpos E, Ntanasis Stathopoulos I, Gavriatopoulou M, Dimopoulos MA. Pathogenesis of bone disease in multiple myeloma: from bench to bedside. Blood Cancer J 2018;98:1-7.

44. Rousseau JC, Sornay Rendu E, Bertholon C, Chapurlat R, Garnero P. Serum periostin is associated with fracture risk in postmenopausal women: a 7-year prospective analysis of the OFELY study. J Clin Endocrinol Metab 2014;99:2533-9.

45. Anastasilakis AD, Polyzos SA, Makras P, Savvides M, Sakellariou GT, Gkiomisi A, et al. Circulating periostin levels do not differ between postmenopausal women with normal and low bone mass and are not affected by zoledronic acid treatment. Horm Metab Res 2014;46:145-9.

46. Hu F, Shang XF, Wang W, Jiang W, Fang C, Tan D, et al. Highlevel expression of periostin is significantly correlated with tumor angiogenesis and poor prognosis in osteosarcoma. Int J Exp Pathol 2016;97:86-92. 